\title{
The Observational Approach to Cosmology
}

GlYCE its first announcement by Dr. E. P. $D$ Hubble in 1929, the existence of a linear relation between 'velocity' and distance among the extra-galactic nebulæ has excited a great deal of theoretical interest, the more so since it appeared to be a direct confirmation of the instability of the Einstein universe predicted by Lemaitre. It was therefore not unfitting that the discoverer of the relation, himself a Rhodes scholar, should have been invited to deliver the Rhodes Memorial Lectures in Oxford on October 29 and November 12 and 26, or that he should have used this opportunity to present the observer's interpretation of the wealth of material since accumulated on this problem by himself. The lectures, which dealt in turn with the observable region, the role of the red-shifts, and possible models of the universe, have revealed that a static universe with a hitherto unsuspected dependence of light frequency on distance is probably more acceptable than one or other of the homogeneous expanding models of general relativity.

This startling conclusion depends in a very large measure on the 'luminosity' methods of distance determination necessarily used for the extragalactic nebulæ. The intrinsic luminosity of the average extra galactic nebula having been determined by means of the Cepheid variables found in nebulæ of the 'local group', the distance of any nebula can be found from its apparent luminosity and the inverse square law. The apparent luminosity is, however, affected by the observed redward displacement of the spectrum. Assuming that the energy distribution in the spectrum of an extra-galactic nebula is similar to that of the sun, as is very nearly the case, and correcting for atmospheric absorption, reflectivity of the telescope and plate sensitivity, one can find empirically the correction, $\Delta m_{1}$, to be applied to the apparent photographic magnitude for any desired red-shift; with sufficient accuracy this correction is given by $\Delta m_{1}=3 \Delta \lambda / \lambda$. If, however, the nebulæ are actually receding from us, an energy correction alone will not suffice, but in addition a correction must be made for the fact that we receive fewer quanta per second from a receding nebula than from a stationary one. This correction amounts to $\Delta m_{2}=\Delta \lambda / \lambda$. Clearly the apparent luminosity to be used in finding the distance of the nebula from its intrinsic luminosity and the inverse square law will depend upon whether the nebula is receding or not, and if there were only some independent means of distance determination the presence or absence of recession could be immediately inferred. Even in the absence of an independent method of distance determination, it is still possible to make some progress in distinguishing between recession and non-recession by counts of nebulæ.

If the total number of nebulæ, or the number of nebulæ per square degree, are counted to different limiting apparent magnitudes, and these limiting magnitudes after the appropriate corrections for recession or non-recession converted to distances, the nebular counts will yield immediately the spatial distribution of nebulæ as a function of distance. In his recent lectures, Hubble used five nebular counts to limiting apparent magnitudes $18 \cdot 5,19 \cdot 0,19 \cdot 4,20 \cdot 0$ and $21 \cdot 0$, the second of these counts having been made by Mayall with the 36 -in. Crossley, the remainder by Hubble with the 60 -in. and the 100 -in.; the last count, it is interesting to note, is within half a magnitude of the limit set for the 100-in. by scattered sky light. Correcting these limiting magnitudes for energy effect alone (no recession), the five counts are perfectly represented by the relation

$$
\log N(m)=0 \cdot 6\left(m-\Delta m_{1}\right)+\text { constant. }
$$

This linear relation is precisely that which would hold if the spatial distribution of the nebulæ were uniform out to the limit of the counts, that is, to a distance of some five hundred million light years. We can interpret this result to mean that we are seeing only a portion of a much larger universe, a portion within which the spatial distribution of nebulæ is uniform, and a universe in which the frequency of a light quantum varies linearly with the distance. Without in any way straining the observations, but at the expense of a newly postulated property of radiation, we can describe the nebular counts in terms of a simple static universe.

If, on the other hand, the limiting magnitudes of the nebular counts are corrected for recession as well as for energy, the resulting apparent spatial distribution is one centrally symmetrical about the galactic system with necessarily increasing spatial density outwards. Since our galactic system appears to be closely similar in intrinsic luminosity and mass to the nebulæ whose density distribution we are discussing, there is no reason why the galactic system should occupy so dominant a position in the universe, and we must regard this spatial distribution, found when we assume the nebulæ to be receding, as only apparent. On 
Milne's original kinematic model of the universe, this outward increase in density is just what would be expected from the fact that we do not have, as it were, an instantaneous view of the universe, but are seeing the more distant nebulæ as they were some five hundred million years ago. When the observed density distribution is corrected to the instantaneous view, the resulting spatial dis. tribution turns out to be uniform; since on the kinematic model, however, uniform density distribution can only occur once, Hubble finds it nearly as unsatisfactory to suppose that we are just happening to observe the universe at a special instant in time, as if we were observing from a specially favoured point in space.

Still assuming that the observed red displacement is due to a velocity of recession, and that therefore the apparent spatial distribution of nebulæ increases radially outwards, Hubble next examined the apparent distribution of nebulæ predicted by the homogeneous expanding models of general relativity, a special case of which also includes in many of its aspects Milne's kinematic model. Tolman has shown that the nebular counts predicted by these models take the form

$$
\log N(m)=0 \cdot 6\left\{m-\Delta m_{1}-\Delta m_{2}+F(R)\right\}
$$

where $R$ is the radius of space curvature. At the very outset an almost insuperable difficulty is encountered in that the observed counts do not obey a relation of this form. If, however, it be assumed that the observed counts are subject to almost impossibly large systematic errors, the relation and the observations may be used to calculate a value of $R$. The resulting value is of the order of five hundred million light years, rather less than the penetrating power of the 100 -in. telescope, and since there is a relation between $R$ and the density of matter in space, the density must be $10^{-26} \mathrm{gm}$. $\mathrm{cm}^{-3}$, which is many times greater than the mean density produced by the nebulæ alone. To account for this density we must postulate that inter-nebular space is filled with matter the mass of which is greatly in excess of the total mass of the nebulæ, and which is distributed in such a form as to produce no absorption of light in space, since any absorption would demand a still smaller radius of curvature and larger mean density.

If no recession is assumed, the observed nebular counts are satisfactorily described by supposing that we are observing a finite portion of a much larger universe of nebulæ, but a universe in which the frequency of light varies uniformly with the distance. If, on the other hand, recession is assumed, the observed nebular counts are not satisfactorily described by any of the homogeneous expanding models of general relativity, but if forced to fit require that the universe be closed, that we have already explored it to its outmost bounds with the 100-in. telescope, and that it is a universe dominantly filled with non-luminous matter distributed in such a way as to absorb or scatter negligibly small amounts of light.

The large and appreciative audiences who followed the three lectures, each a model of exposition and clarity, had little difficulty in agreeing with Dr. Hubble that the consequences of assuming no recession were the less difficult to accept.

H. H. P.

\section{News and Views}

\section{Prof. A. N. 'Talbot}

Prof. Arthur N. TAlbot, emeritus professor of engineering in the University of Illinois, has been awarded the John Fritz Gold Medal, the highest of American engineering honours. Prof. Talbot, who is seventy-nine years of age, was cited as "moulder of men, eminent consultant on engineering projects, leader of research, and outstanding educator in civil engineering". The award is made annually by a board composed of sixteen past-presidents of the four national societies of civil, mining and metallurgical, mechanical and electrical engineers. Prof. Talbot was born in Cortland, Ill., on October 21, 3857. He has been engaged in engineering work since 1881 , his activities embracing railroads, roads, bridges, buildings and municipal public works. Prof. Talbot aided in the development of the testing laboratories and the College of Engineering of the
University of Illinois. He has been active in the formation and development of the Illinois Engineering Experiment Station, in connexion with which he ha's made numerous investigations in the properties of steel, brick, concrete and reinforced concrete, and in water purification, sewage treatment and hydrau. lics. Among previous recipients of the John Fritz Gold Medal have been Lord Kelvin, Thomas Edison, Guglielmo Marconi, Elihu Thomson and Sir Robert Hadfield.

\section{Sir Robert Mond}

Srr ROBERT Mond has been elected an associate foreign member of the Académie des Inscriptions et Belles Lettres in Paris, in succession to the late King Fuad of Egypt. Sir Robert Mond's wide interests are illustrated by the fact that he is honorary secretary of the Davy Faraday Research Laboratory at the 